\title{
The genetic component of the human embryonic selection: uncovering of the strength and main targets
}

\author{
Sergey Oreshkov \\ Center for Mitochondrial Functional \\ Genomics, IKBFU, Kaliningrad, Russia \\ s.oreshkov@live.com \\ Dmitrii Iliushchenko \\ Center for Mitochondrial Functional \\ Genomics, Immanuel Kant Baltic \\ Federal University \\ Kaliningrad, Russian Federation \\ iliushchenkodmitrii@gmail.com
}

\author{
Elisaveta Zezyulya \\ Center for Mitochondrial Functional \\ Genomics, IKBFU, Kaliningrad, Russia \\ liza.zez1@hotmail.com \\ Evgenii Tretiakov \\ Center for Brain Research, Medical \\ University of Vienna \\ Vienna, Austria \\ Orcid 0000-0001-5920-2190
}

\author{
Konstantin Gunbin \\ Institute of Cytology and Genetics SB \\ RAS, Novosibirsk, Russia \\ Center for Mitochondrial Functional \\ Genomics \\ IKBFU, Kaliningrad, Russia \\ genkvg@gmail.com \\ Konstantin Popadin \\ School of Life Sciences \\ Ecole Polytechnique Federale de \\ Lausanne, Lausanne, Switzerland \\ Center for Mitochondrial Functional \\ Genomics, IKBFU, Kaliningrad, Russia \\ konstantinpopadin@gmail.com
}

\begin{abstract}
Runs of Homozygosity(ROH) are sites in the two homologous chromosomes that are identical by descent in an individual. Excess of ROHs carries a risk associated with various diseases. At the same time, long ROHs in healthy individuals are enriched with slightly deleterious variants, which are constantly filtered out by selection. Hence, distribution and length of Runs of Homozygosity is affected by recombination and purifying selection. Data from families with different levels of consanguinity allows us to access both these effects and their influence on highly autozygoteous pedigree. Understanding connections between runs of homozygosity, recombination and purifying selection will help us to better understand evolutionary processes in highly inbred populations.
\end{abstract}

Keywords - Runs of Homozygosity, consanguinity, recombination, purifying selection, in-silico modeling

\section{Introduction}

Human embryonic selection is extremely poorly known since the majority of miscarriages occur very early and there is no possibility to collect a representative dataset of miscarried samples for consequent genetic analyses. All such collections will be (i) biased towards late miscarriages and (ii) will not be able to shed light on potential selection during germline differentiation: formation of oocytes and spermatozoids. The best approach to estimate the strength and targets of human embryonic selection is to compare parental and offspring genomes (hereafter "trios") in order to uncover over- or under- inherited alleles. It is rational to start to develop and test tools dissecting the transmission disequilibrium from parents to offspring using collection of trios with expected strong miscarriage rate - such as consanguineous families.

\section{Methods}

Consanguinity increases the risk of expression of deleterious autosomal recessive mutations causing a correlation between the coefficient of inbreeding, F [1], and the probability of offspring to survive. The more closely related the parents, the higher the mortality of the children [2]. Importantly, the nominal coefficient of inbreeding, F, derived from pedigree, approximates an expected fraction of loci, identical by descent, however the observed coefficient of inbreeding in each particular case, Fo, can significantly fluctuate between full siblings due to stochasticity in the number and position of recombination events during meiosis as well as random gamete fusion. For example, for siblings from a first cousin marriage, the nominal coefficient of inbreeding is $0.0625(1 / 16)$, however its standard deviation is very high (0.0243), causing great variation in probability of survival. Thus, variation in $\mathrm{F}$ provides a material for selection inside each family and we expect that Fo (healthy siblings) < Fo (affected siblings) $<$ Fo (expected siblings).

As a molecular metric approximating the observed coefficient of inbreeding, Fo, we use the portion of genome covered by long Runs Of Homozygosity (ROHs) [3]. Runs of homozygosity $(\mathrm{ROH})$ - stretches of the two homologous chromosomes within the same individual that are identical by descent. Long $\mathrm{ROH}$ are enriched in deleterious variants in healthy individuals[4] and an excess of ROHs is a risk factor for Parkinson disease, Alzheimer disease, Schizophrenia, speech delay and autism and the degree of intellectual disability. This implies that long ROHs carry one severe or many mildly-deleterious recessive alleles and thus there is a selection force against long ROHs in the human population.

\section{Datasets}

Main dataset is genotype data of 50 consanguineous families from the study of Makrythanasis et al. [5]. Additionally, there is information about pedigree(the tree) and exomes of affected and unaffected siblings.

All families have at least one unaffected offspring and at least two individuals with intellectual disability or development delay.

\section{Results}

Using our main dataset we have performed our insilico pipeline and compared the total length of long ROHs in healthy versus affected (with intellectual disabilities, ID) and expected (in-silico generated) siblings. For in-silico kids derived randomized models for expected siblings, which captures distribution properties of heterozigotious recombination in offspring. We produced several models of synthetic offspring, which capture different aspects of the source dataset. In different models we capture 
recombination and selection features such as different distribution of $\mathrm{ROHs}$ and different recombination rate. We have observed that healthy siblings are indeed characterized by the decreased total length of ROHs as compared to affected. Additionally we have demonstrated an excess of $\mathrm{ROH}$ in expected (generated in silico) versus born siblings (both affected and unaffected). Altogether it suggests an action of purifying selection against long ROHs.

\section{Discussion}

In this project we concentrated on the estimation of the strength of the short-term selection against the autozygome (the fraction of the genome that has been biparentally inherited from the same ancestor). For this, we compared the fractions of autozygous regions in genomes of unaffected, affected and theoretically expected (in silico modeled) siblings in each consanguineous family. Selection against autozygous regions manifested itself as a decrease of this fraction in unaffected versus affected and expected siblings.

The strength of the decrease can depend on phenotypes of the affected offspring (Mendelian or complex disease) as well as the level of consanguinity of parents and the previous history of the parental consanguinity. We conclude that this approach is effective in uncovering the strength and targets of human embryonic selection and plan to extend it towards analysis of polygenic risk scores (PRS) of parents and offspring as well as analysis of new human trios.

\section{REFERENCES}

[1] Wright S., "Coefficients of inbreeding and relationship" The American Naturalist 56, pp 330-338, 1922.

[2] Newton E. Morton, James F. Crow, and H. J. MullerJ. Clerk Maxwell, "An estimate of the mutational damage in man from data in consanguineous marriages" PNAS, November 1, 42(11), 1956, pp.855-863.

[3] McQuillan R et al. "Runs of homozygosity in European populations." Am J Hum Genet. Sep;83(3), 2008, pp 359-72.

[4] Szpiech ZA at al. "Long runs of homozygosity are enriched for deleterious variation.” Am J Hum Genet. Jul 11;93(1), 2013 , pp. 90-102.

[5] Makrythanasis P., et al. "Diagnostic exome sequencing to elucidate the genetic basis of likely recessive disorders in consanguineous families.” Hum Mutat. Oct;35(10), 2014 , pp. $1203-10$ 